\title{
Flying particle sensors in hollow-core photonic crystal fibre
}

D. S. Bykov*, O. A. Schmidt, T. G. Euser* and P. St.J. Russell

Max Planck Institute for the Science of Light, Guenther-Scharowsky-Str. 1, 91058 Erlangen, Germany *e-mail: dmitry.bykov@mpl.mpg.de, tijmen.euser@mpl.mpg.de

Optical fibre sensors make use of diverse physical effects to measure parameters such as strain, temperature and electric field. Here we introduce a new class of reconfigurable fibre sensor, based on a 'flying-particle' optically trapped inside a hollow-core photonic crystal fibre (HC-PCF), and illustrate its use in electric field and temperature sensing with high spatial resolution. The electric field distribution near the surface of a multi-element electrode is measured with a resolution of $\sim 100$ microns by monitoring changes in the transmitted light signal due to the transverse displacement of a charged silica microparticle trapped within the hollow core. Doppler-based velocity measurements are used to map the gas viscosity, and thus the temperature, along a HC-PCF. The flying-particle approach represents a new paradigm in fibre sensors, potentially allowing multiple physical quantities to be mapped with high positional accuracy over kilometer-scale distances.

Their flexibility, compactness and immunity to unwanted electromagnetic interference has made fibreoptic sensors, based on conventional step-index fibres, attractive in many contexts ${ }^{1}$. Fibre Bragg gratings are routinely used to monitor changes in strain or temperature ${ }^{2-5}$, and if the fibre is coated with magnetostrictive materials, magnetic fields can be sensed ${ }^{5,6}$. The linear and nonlinear optical properties of the glass core have been used to measure temperature ${ }^{1,3}$ and magnetic field ${ }^{7-9}$ and radiation-induced photo-darkening or luminescence is used in radiation dosimetry ${ }^{10}$. Systems using optical time domain reflectometry can reach a spatial resolution of $\sim 1 \mathrm{~cm}$ in strain and temperature sensing $^{3}$, while resolutions of $10 \mu \mathrm{m}$ have been reached in frequency-domain optical backscattering schemes $^{11}$.

In a non-fibre context, micrometre-scale spatial resolution can be obtained by detecting the displacement or rotational speed of an optically tweezered microparticle ${ }^{12}$. This has been used to measure pN-scale external forces ${ }^{13}$, the viscosity of liquids ${ }^{14,15}$ and gases ${ }^{16}$ and (using charged 
spheres) the electric field near microelectrodes in a liquid environment ${ }^{17}$. The probe area must however lie within the field-of-view of a bulky high-resolution microscope, limiting the usefulness of this approach. The range over which microparticles can be positioned can be extended by optically propelling them inside capillary fibres; however, high transmission loss limits the propulsion length to a few $\mathrm{cm}$ at most ${ }^{18}$. Hollow-core photonic crystal fibre (HC-PCF) offers much lower optical loss, allowing microparticles to be positioned to micrometre precision over long distances, limited only by the fibre loss ${ }^{19-23}$. PCF has also been used in a range of other sensing applications ${ }^{24,25}$.

Here we present a new class of sensor based on a microparticle optically trapped and propelled inside the core of a low-loss HC-PCF. A variety of different types of particle-environment interaction, or particle materials, can be used to map multiple physical quantities with high spatial resolution along a single fibre line. The surrounding glass cladding shields the transducer particle from unwanted external influences such as gas flow or chemical attack, potentially enabling measurements to be made even in harsh or challenging environments. 
(a)
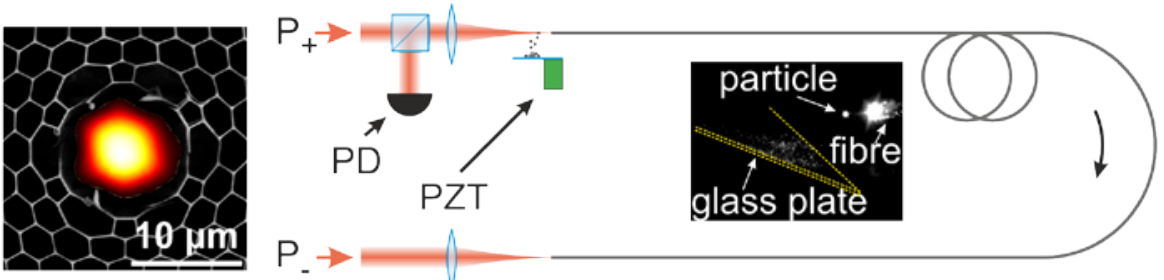

(b)

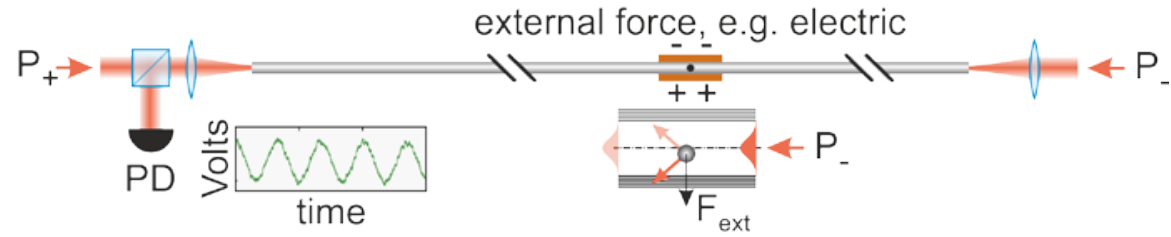

(c)

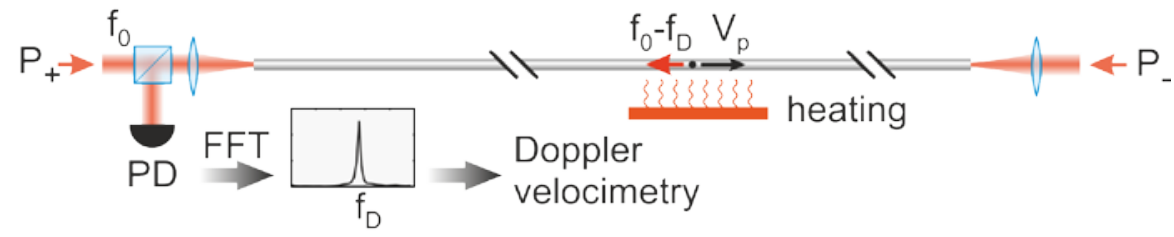

(d)

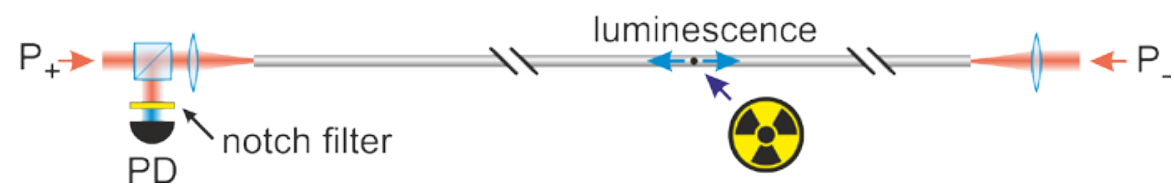

Figure 1| Schematic of measurement procedures. a, Measurement set-up and scanning electron micrograph of a HC-PCF (core diameter $12 \mu \mathrm{m}$ ) with a superimposed near-field optical mode profile (at $1064 \mathrm{~nm}$ wavelength). A particle is initially trapped in front of the PCF. By adjusting the power in the forward and backward propagating beams $\left(\mathrm{P}_{+}, \mathrm{P}_{-}\right)$the particle can be moved along the fibre or held stationary. The transmitted power is monitored using a photodiode (PD). Different types of particleenvironment interaction can occur in the measurement region: $\mathbf{b}$, An external force acting on the particle, $\mathbf{c}$, a change in the environmental conditions, for example the temperature, or $\mathbf{d}$, a change in particle state, for example, radioluminescence.

A microparticle is first launched into the HC-PCF (Figure 1a) and its position controlled by adjusting the power in the counter-propagating optical modes (see methods). Once in the detection zone, its interaction with the environment is remotely probed by monitoring the transmitted and reflected light. Several different sensing schemes are possible. If for example the whole fibre is subject to transverse mechanical vibration or linear acceleration, the particle will be perturbed from its trapped position, causing a change in the transmitted optical signal (Figure. 1b). If the particle has charge $q$, displacements caused by electric $(q \mathbf{E})$ and magnetic $\left(q \mathbf{v}_{\mathrm{p}} \times \mathbf{B}\right)$ forces can be measured. If the particle is itself magnetised, the local magnetic field could be monitored even for a stationary particle. Another 
sensor type makes use of the fact that the particle velocity is inversely proportional to the viscosity of the gas (or liquid) filling the core, which is itself dependent on the temperature (Figure 1c). Doppler velocimetry can be used to measure the temperature with very high spatial resolution. In yet another scenario, radio-luminescent or radiochromic particles could be used to monitor radiation levels in the local environment ${ }^{10}$ (Figure 1d). Such microdosimeters could be held at specific locations along the fibre, their optical properties being remotely monitored via the waveguide modes. If a fluorescent particle is used, the local temperature could be monitored via changes in the luminescence intensity or lifetime.

Here we report the results on electric field and viscosity-based temperature sensing, using silica microspheres as particles. In the first experiment, a length of HC-PCF was placed between two planar electrodes, so as to generate an electric field $E$ perpendicular to the fibre axis. A silica particle (radius $3 \mu \mathrm{m}$ ), randomly charged by triboelectric effects, was launched into a HC-PCF with core diameter 7.8 $\mu \mathrm{m}$ and trapped between the electrodes at a fixed position. A pulsed laser (Ti:sapphire, $800 \mathrm{~nm}$ ) was used for the trapping beams, so as to reduce the effects of intermodal beating and maximize the homogeneity of the optical potential along the fibre (see Methods). A sinusoidally modulated voltage was then applied to the electrodes, causing the particle to oscillate about its trapping position, thus altering the fraction of light scattered by the particle and modulating the transmitted signal (Figure 1b).

The trapped particle may be treated as a damped harmonic oscillator. For small displacements $x$ from the trapping position the optical restoring force takes the form $F_{x}=-k x$, where the spring constant $k$ is proportional to the optical power ${ }^{26}$. The transfer function between the sinusoidal driving amplitude $\bar{E}(\omega)$ at frequency $\omega$ and the response $\bar{x}(\omega)$ may be written in the standard form:

$$
\bar{x}(\omega)=\frac{q / m}{\sqrt{\left(\omega^{2}-\omega_{0}^{2}\right)^{2}+4 \gamma^{2} \omega^{2}}} \exp \left(i \arctan \left(\frac{2 \gamma \omega}{\omega^{2}-\omega_{0}^{2}}\right)\right) \bar{E}(\omega)
$$

where $\omega_{0}=\sqrt{k / m}, \gamma=K 6 \pi a \mu / m$ is the damping rate, $m$ the particle mass and $a$ its radius, and $\mu$ the viscosity of air. $K$ is a correction factor that takes into account the additional drag force due to 
confinement of the particle in a narrow cylindrical channel. Note that the damping rate can be reduced by lowering the air pressure (this is done by enclosing both fibre ends in windowed gas cells connected to a vacuum pump), via the pressure-dependent viscosity, which follows the relationship ${ }^{27}$ :

$$
\mu(p)=\frac{\mu_{0}}{1+K n(p)\left(\beta_{1}+\beta_{2} e^{-\beta_{3} / K n}\right)}
$$

where (for air) $\mu_{0}=1.81 \times 10^{-5} \mathrm{~Pa} \cdot \mathrm{s}, \boldsymbol{\beta}_{1}=1.231, \boldsymbol{\beta}_{2}=0.469$ and $\boldsymbol{\beta}_{3}=1.178$. The Knudsen number $\mathrm{Kn}$ $=\Lambda / L_{\mathrm{c}}$ where $\Lambda=k_{\mathrm{B}} T /\left(\sqrt{2} \pi d^{2} p\right)$ is the mean free path, $L_{\mathrm{c}}$ is the characteristic dimension of the system (see below), $k_{\mathrm{B}}$ is Boltzman's constant, $T$ the temperature, $p$ the pressure in Pascal and $d=$ $3.6 \times 10^{-10} \mathrm{~m}$ is the collision diameter of the molecules. The resulting damping rate at atmospheric pressure is $\gamma=3.2 \mathrm{kHz}$, reducing by one order of magnitude at a pressure of 10 mbar.

Figure 2a shows the measured frequency response for a particle of diameter $3 \mu \mathrm{m}$ trapped by $22 \mathrm{~mW}$ (11 mW in each direction) of guided optical power in a HC-PCF at a pressure of 14 mbar. The data shows a clear harmonic oscillator response in phase and amplitude, with a resonant frequency of 3.3 $\mathrm{kHz}$. The modulation of transmitted optical power depends on $x(t)$ in a generally nonlinear way, being caused by scattering into high-loss higher-order core and cladding modes. Since clearly the dominant response will at the second harmonic of the driving electrical signal, good fits to the experimental data are obtained if the signal is represented as:

$$
S_{\text {opt }}(t)=\left(a_{2}|\bar{x}(\omega)|^{2}+a_{4}|\bar{x}(\omega)|^{4}\right) \cos (2 \omega t+\phi)
$$

where the constants $a_{n}$ are chosen to give the best fit to the data and the phase relative to the driving electric signal is $\phi=\arctan \left(4 \gamma \omega /\left(\omega^{2}-\omega_{0}^{2}\right)\right)$. Since there is a linear relationship between the amplitudes of the applied electric field and the mechanical oscillations (equation (1)), the system can be calibrated by measuring the amplitude of the optical signal at $2 \omega$ for a range of different electric field amplitudes (Figure 2a, inset). A good fit was obtained for $a_{2}=1.5(\mathrm{~cm} / \mathrm{kV})^{2}$ and $a_{4}=0.04$ $(\mathrm{cm} / \mathrm{kV})^{4}$. The resulting theoretical curves are in excellent agreement with the measured data for a resonant frequency of $3.3 \mathrm{kHz}$ and a damping rate of $560 \mathrm{~Hz}$. 
The damping rates, measured for pressures between 4 and 100 mbar (Figure 2b), are in very good agreement with the viscosity expected from the Cunningham correction factor (equation (2)). This enabled us to extract the characteristic size of the system as $L_{\mathrm{c}}=1.2 \mu \mathrm{m}$ and the drag correction factor as $K=2.5$. This value of $K$ is comparable with the previously reported value (2.8) for particles of a similar size, moving perpendicular to a single flat interface ${ }^{28}$.

(a)

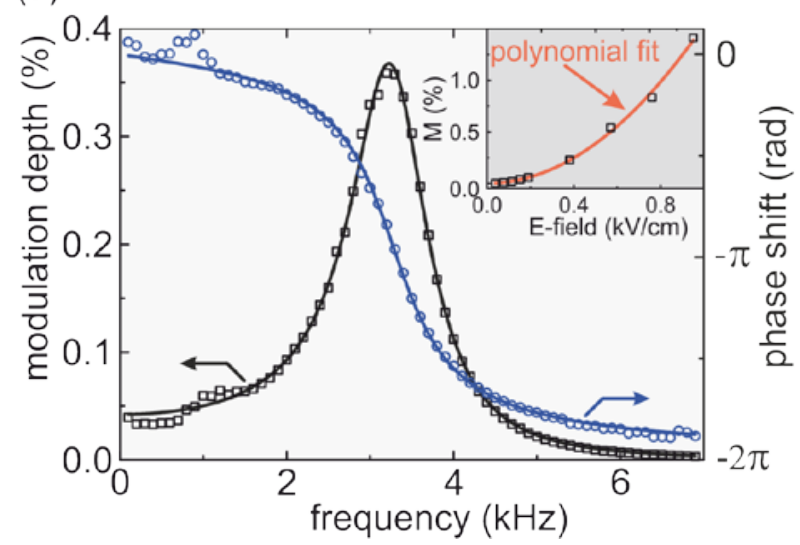

(b)

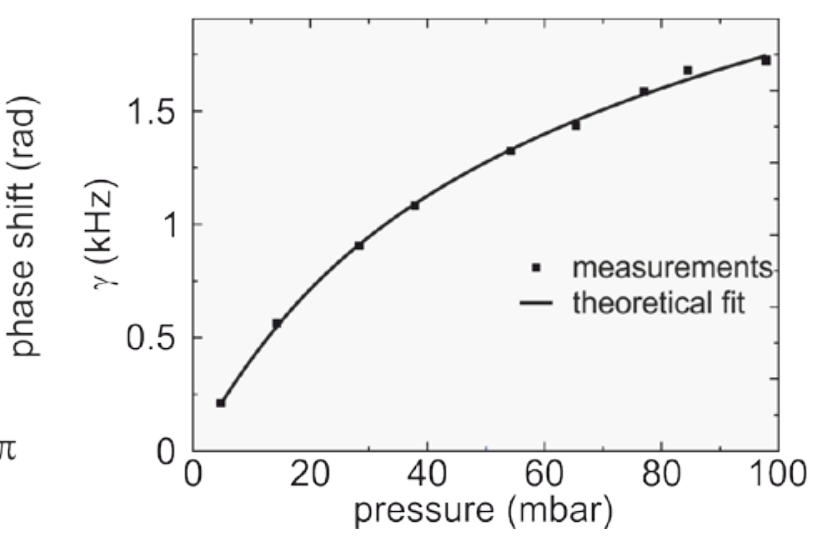

Figure 2| Measurements of the particle response at different pressures. a, Typical frequency response of the second harmonic signal plotted versus applied electric field frequency for a particle trapped in HC-PCF and driven by an external electric field (14 mbar, $22 \mathrm{~mW}, 760 \mathrm{~V} / \mathrm{cm})$. The dots are experimental datapoints and the full curves fits to the theory. The inset shows a calibration curve at 6.2 mbar and $22 \mathrm{~mW}$ ( $M$ is the modulation depth). b, Damping coefficients extracted from the theoretical fits, plotted against pressure. The full curve is a theoretical fit.

The resonant frequency was next measured at a constant pressure of 6.5 mbar for optical powers between 6 and $27 \mathrm{~mW}$. Two typical response curves are plotted in Figure 3, and show excellent agreement with theory, the resonant frequency shifting from $3.3 \mathrm{kHz}$ (at $26.6 \mathrm{~mW}$ ) to $2.9 \mathrm{kHz}$ (at 21.2 $\mathrm{mW})$. As expected at constant pressure, the damping rates are almost identical in both measurements (254 and $256 \mathrm{~Hz}$ ). The inset in Figure 3 shows that the square of the resonant frequency is proportional to the optical power, as expected if the spring constant $k$ scales linearly with optical power. The results demonstrate that the frequency response of the detector can be optimized for specific measurement tasks simply by changing the stiffness of the optical trap. 


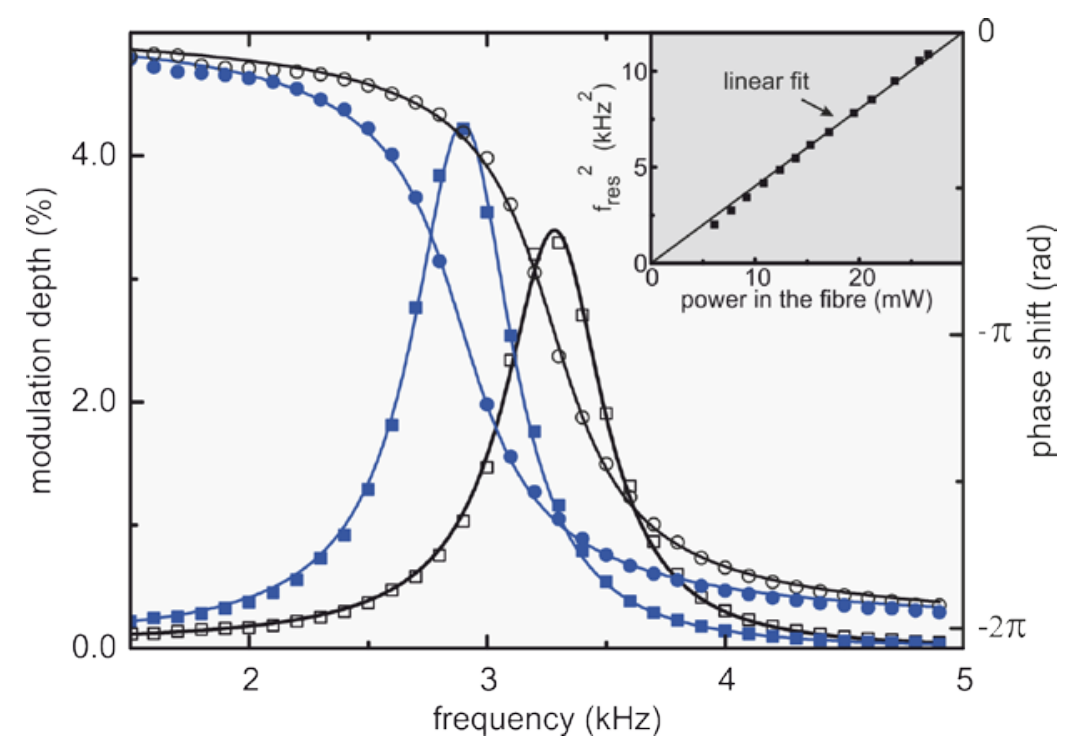

Figure 3| Measurement of the particle response at different powers. Frequency spectrum of the response at 6.5 mbar pressure plotted versus applied electric field frequency for two different optical powers (21.2 mW, blue curve with filled-in symbols; $26.6 \mathrm{~mW}$, black curve with open symbols). The applied electric field amplitude was $95 \mathrm{~V} / \mathrm{cm}$. Inset: scaling of the square of the measured resonant frequency $\left(f_{\text {res }}\right)$ with the total optical power at the particle position. The slope is $0.4 \mathrm{kHz}^{2} / \mathrm{mW}$.

To demonstrate spatially resolved sensing, a position-dependent electric field was created by placing part of the fibre between two electrodes (Figure 4a), one of which consisted of a pattern of metallic stripes of different width, the smallest being $200 \mu \mathrm{m}$ wide (Figure 4b). To ensure constant spacing between the electrodes, two $400 \mu \mathrm{m}$ thick glass slides were used as a spacer. The fibre (outer diameter $110 \mu \mathrm{m}$ ) was placed on the patterned electrode. A sinusoidally modulated voltage (frequency $250 \mathrm{~Hz}$, amplitude $16 \mathrm{~V}$ ) was applied to the system. The expected profile of the transverse electric field in the PCF core was obtained from finite element modelling (COMSOL Multiphysics) and is shown in Figure 4c (upper). The field strength near the centre of each electrode strip is $400 \mathrm{~V} / \mathrm{cm}$, and sharp spikes in electric field appear at the edges of each electrode.

To spatially resolve the electric field, a charged silica microparticle was optically trapped and launched into the fibre. As a first step, the sensor amplitude was calibrated using two unstructured electrodes, located near the fibre entrance. The resonant frequency was then set by adjusting the power in the trapping beams. Next, the particle was propelled along the fibre at a speed of about $1 \mathrm{~mm} / \mathrm{s}$. An additional weak probe laser (830 nm, continuous wave - CW) was used to monitor the particle velocity 
by Doppler velocimetry ${ }^{21}$, while the transmitted intensity of one of the trapping beams was used to monitor the field-induced transverse oscillations. The data was analysed by taking fast Fourier transforms of 50-ms-wide windows (representing 12.5 oscillations of the electric field at $250 \mathrm{~Hz}$ ). Combined with the particle velocity of $1 \mathrm{~mm} / \mathrm{s}$, this yielded a spatial resolution of $50 \mu \mathrm{m}$. A detailed description of the measurement procedure is available in Methods. The measured electric field profile (Figure 4c, lower) is in very good agreement with the calculations. All the features in the calculated field pattern are well-resolved, including the $\sim 100 \mu \mathrm{m}$ wide spikes near the edges of the electrodes. The ultimate spatial resolution is limited only by the microparticle size, which can be as small as 1 $\mu \mathrm{m}$. It would also be possible to enhance the sensitivity by using smaller particles, optimizing the fibre design, or by increasing the particle charge prior to launching into the fibre. 
(a)

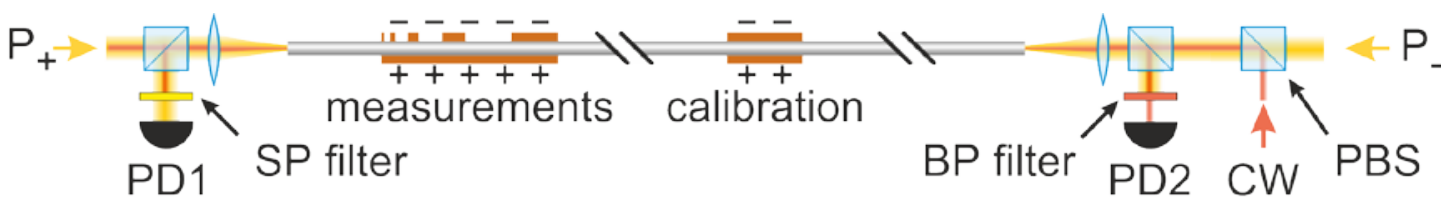

(b)

fibre $\ldots 1 \quad 2 \quad 3 \quad \frac{3}{\text { flying particle }}$

(c)

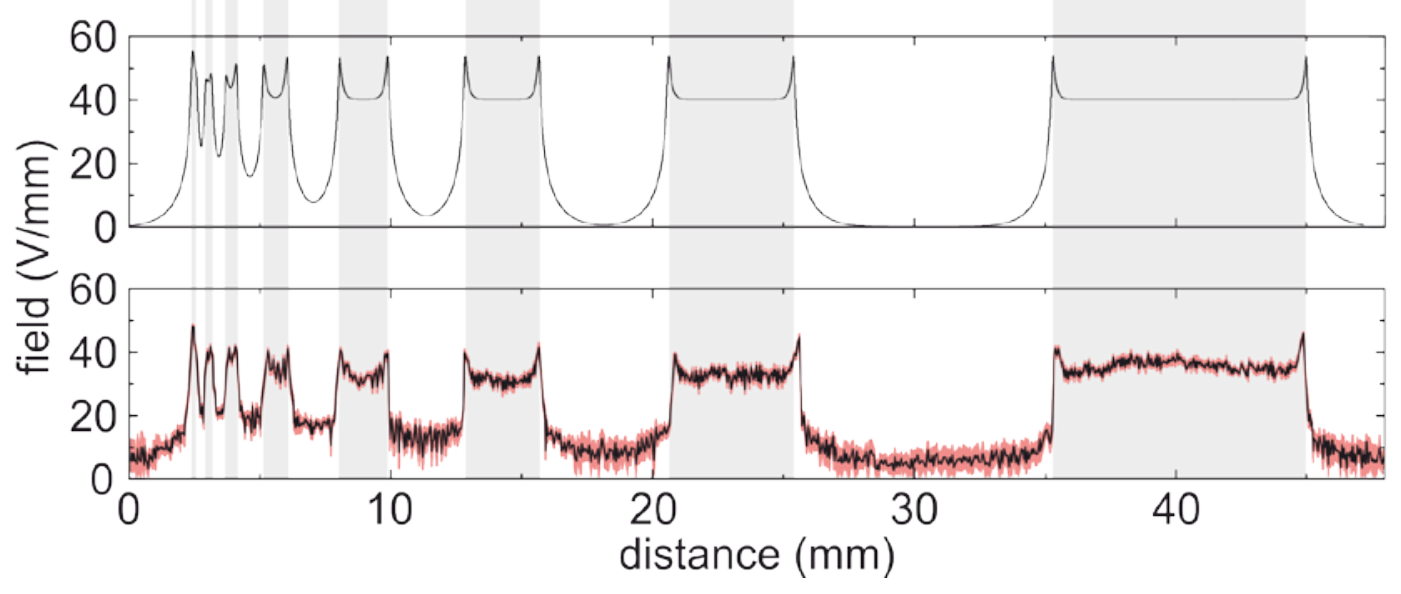

Figure 4| Spatially resolved measurements of the electric field. a, Schematic of the set-up used for spatially-resolved measurements. b, Cross-section of the patterned electrode (not to scale). The smallest three electrodes have widths $0.05,0.1$ and $0.2 \mathrm{~mm}$ and the gap widths are 10, 5, 3, 2, 1, 0.5 and $0.3 \mathrm{~mm}$. c, Field component normal to the fibre axis, calculated by solving the Helmholtz equation (upper) and flying particle measurement of the field (lower). The red shading represents the estimated error, obtained from the experimentally measured noise level in absence of an external electric field.

The flying-particle system can also be used to measure temperature, via the dynamic viscosity $\mu$ which is given by the Sutherland's formula ${ }^{29}$ :

$$
\mu(T)=\mu_{\text {ref }}\left(\frac{T}{T_{\text {ref }}}\right)^{3 / 2} \frac{T_{\text {ref }}+S}{T+S}
$$

where $\mu_{\text {ref }}$ is the viscosity at a reference temperature $T_{\text {ref }}$ and $S$ the Sutherland constant. For air these values are $\mu_{\text {rf }}=1.84 \times 10^{-5} \mathrm{~kg} / \mathrm{m} \cdot \mathrm{s}, T_{\text {ref }}=293 \mathrm{~K}$ and $S=111 \mathrm{~K}^{30}$. The viscosity was measured by monitoring the velocity of a particle optically propelled along an air-filled HC-PCF. In the experiment a CW laser with wavelength $1064 \mathrm{~nm}$ was used (its longer coherence length was need for the Doppler 
measurements) in combination with a HC-PCF of core diameter $12 \mu \mathrm{m}$. The steady-state particle velocity is reached when the viscous and optical forces exactly balance:

$$
F_{\mathrm{opt}}=K 6 \pi a \mu V_{\mathrm{p}}
$$

where $V_{\mathrm{p}}$ is the particle velocity, $a$ the particle radius and $K$ is the correction factor mentioned above 31. To demonstrate spatially-resolved temperature sensing, a silica microparticle (4 $\mu$ m diameter) was optically propelled along 2 m of HC-PCF, while its velocity was monitored by Doppler velocimetry. The first $1.8 \mathrm{~m}$ of fibre was kept at room temperature $\left(T_{\text {ref }}=293 \mathrm{~K}\right.$ ) and used as a reference. A $20 \mathrm{~cm}$ length of the fibre was passed into a temperature-stabilized furnace. The resulting speed trace for a furnace temperature of $614 \mathrm{~K}$ is shown as a blue curve in Figure 5a,b. The data shows a 40\% drop in velocity as the particle enters the heated furnace, as expected from the temperature-related viscosity increase. The velocity trace also reveals a periodic speed fluctuation with $20 \%$ modulation depth along the entire fibre.

These fluctuations are caused by beating with higher order modes (unavoidably excited - see Methods) and currently limit the spatial resolution to a few $\mathrm{cm}^{21}$. To reduce this effect, a moving average of the speed trace was taken over a 2-cm-wide window (dashed red curve). The experiment was repeated at a range of different temperatures. Figure. 5c shows that the temperature obtained from each speed measurement indeed matches the set temperature. Importantly, for constant optical power, the measured change in particle speed depends on the ratio $\mu_{\text {ref }} / \mu$ only. As a result, the temperature profile can be obtained without knowing the refractive index or diameter of the particle, or the wall correction factor $K$, resulting in a self-calibrated sensing scheme. 
(a)

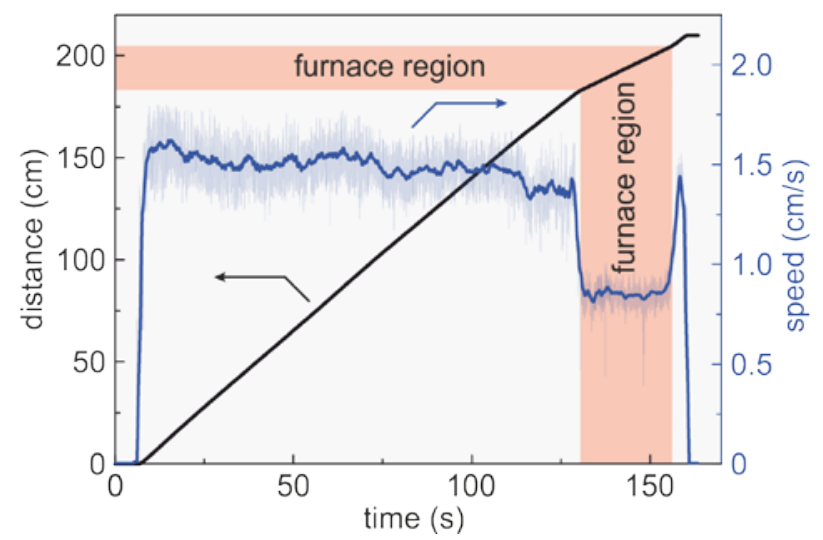

(b)

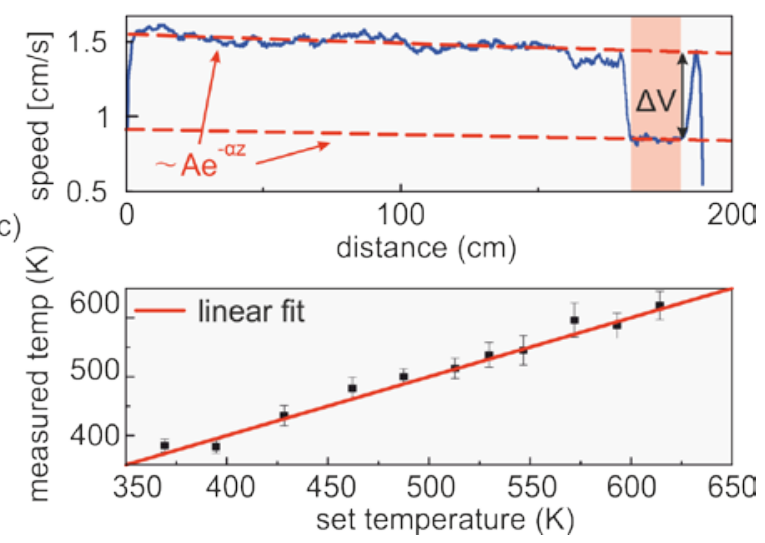

Figure 5| Temperature measurements. a, Position-time plot (black line) of the particle as it passes through the reference region $\left(T_{\text {ref }}=293 \mathrm{~K}\right.$ ) and the furnace (at $614 \mathrm{~K}$ ). The velocity at each point (measured by Doppler velocimetry) is also plotted (light blue curve: the fluctuations are caused by intermodal beating - see text). The solid blue curve is the speed averaged over a moving window 1.3 seconds wide. b, Smoothed speed profile (moving average over a 2-cm-wide window) of the particle with exponential fits (dashed red curves). c, Measured temperature versus set temperature. Error bars were obtained from the standard deviation of the measured microparticle speed.

\section{Conclusions and outlook}

Flying-particle sensors, based on microparticles optically guided along HC-PCF, represent a new paradigm in reconfigurable fibre sensors, potentially allowing multiple physical quantities to be mapped with very high positional accuracy over long distances. The maximum length over which particles can be trapped is limited by the input laser power and the fibre loss. In the experiments reported here, the losses were $80 \mathrm{~dB} / \mathrm{km}$ (for the $1064 \mathrm{~nm}$ fibre), resulting in a maximum manipulation length of $\sim 400 \mathrm{~m}$, assuming a maximum laser power of $10 \mathrm{~W}$. This could be extended to tens of $\mathrm{km}$ if low-loss HC-PCFs are used (the best HC-PCFs have losses of $\sim 1 \mathrm{~dB} / \mathrm{km}$ at $1550 \mathrm{~nm}^{32}$ ). The motion of the trapped microparticle could be also stabilised by spinning it in a circularly polarized laser beam ${ }^{33}$ or by using parametric feedback ${ }^{34}$. The flying particle system could be readily extended to measure other physical quantities such as vibration, magnetic fields and ionizing radiation, all of which can be detected remotely through the waveguide modes. The system uniquely allows high-resolution, multiparameter sensing in a single length of fibre and is of particular interest for use in highly 
radioactive environments (e.g., inside a nuclear reactor) where conventional solid-glass cores rapidly darken due to radiation damage.

\section{Methods:}

\section{Particle manipulation in HC-PCF}

A schematic of the particle sensing set-up is shown in Figure 1a. A laser beam is adjustably split into two beams (power levels $\mathrm{P}_{+}$and $\mathrm{P}_{-}$), which are then launched into opposite ends of the HC-PCF. Two different laser-plus-fibre combinations were used. In the first, a CW 1064 nm laser was used along with a HC-PCF of core diameter $12 \mu \mathrm{m}$. The long coherence length of the laser allowed Doppler velocimetry to be used to monitor particle velocity, although with the drawback that intermodal beating perturbed the particle velocity (excitation of higher order modes was very difficult to eliminate experimentally). In the second, a Ti-sapphire laser ( $800 \mathrm{~nm}, \sim 7$ ps chirped pulses, $30 \mathrm{~nm}$ bandwidth, $80 \mathrm{MHz}$ repetition rate) was used in combination with a HC-PCF of core diameter $7.8 \mu \mathrm{m}$. In freespace the effective trapping potential of a pulsed laser with a sufficiently high repetition-rate is identical to that of a CW laser with the same average optical power ${ }^{35}$. Inside HC-PCF, modal dispersion causes temporal walk-off between pulses propagating in different higher-order modes, effectively eliminating intermodal beating effects. As a result, except for exponential decay due to loss, the transverse trapping potential did not vary along the fibre.

To launch a single particle into the PCF core, it was first held in a dual-beam trap created by the two counter-propagating beams in front of the fibre ${ }^{19}$. To load a particle into the trap, a small quantity of silica microspheres was placed on a glass slide. Particles are detached from the glass plate by driving it at resonance ( $\sim 10 \mathrm{kHz}$ ) using a piezoelectric transducer, until a single particle is optically trapped. The particle can then be moved into and along the fibre by adjusting the power ratio between the two counter-propagating modes.

\section{Microparticle speed and distance measurement}

A fibre-based Doppler velocimetry technique was used to monitor the particle velocity ${ }^{21}$. A small fraction of the light scattered by the moving particle is Doppler-shifted and reflected into the backward 
propagating mode. This light is mixed with unshifted light reflected from the fibre end-face and detected at a photodiode. By monitoring the frequency of the resulting beat-node, the velocity of the particle can be monitored along the entire length of the fibre (within the coherence length of the laser).

\section{Monitoring of the transmitted power}

In the case of a sinusoidally varying external force and perfectly symmetric fibre modes and microparticle we would see a response only at double the drive frequency $\omega$; this is because there is no difference between upwards and downwards particle movement. Asymmetry in the modal pattern (caused, e.g., by higher order modes) or asphericity in particle shape will cause the appearance of hard-to-interpret frequency components at $\omega$. As a result, only the second harmonic amplitude, extracted using an RF spectrum analyser, was used in the experiments.

\section{Fitting the amplitude resonance curves}

To interpret the experimental results, a transfer function between the amplitude of particle oscillation and the transmitted optical power is needed. Since it is clear that this function is likely to be nonlinear, it is appropriate to use a polynomial expansion and write the optical response (the fluctuating intensity at the detector) as follows:

$$
S_{\mathrm{opt}}(t)=\sum_{n} \chi^{(n)} 2^{-n}\left(\bar{x}(\omega) \mathrm{e}^{\mathrm{i} \omega t}+\text { c.c. }\right)^{n}
$$

Since the particle is held in an optical trap, the maximum optical signal is expected to be strongest at twice the frequency of the driving electrical signal. Extracting the second harmonic amplitude from (5):

$$
\bar{S}_{\text {opt }}(2 \omega)=\bar{x}^{2}(\omega)\left(2^{-2} \chi^{(2)}+4 \times 2^{-4} \chi^{(4)}|\bar{x}(\omega)|^{2}+\ldots\right)
$$

which yields a temporal response of:

$$
S_{\mathrm{opt}}(t)=\frac{1}{2}\left(\chi^{(2)}+\chi^{(4)}|\bar{x}(\omega)|^{2}+\ldots\right)|\bar{x}(\omega)|^{2} \cos (2 \omega t+\phi)
$$

where $\phi=2 \arg \bar{x}$. Writing with redefined constants:

$$
\left.S_{\mathrm{opt}}(t)=\left(a_{2}|\bar{x}(\omega)|^{2}+a_{4} \mid \bar{x}(\omega)\right)^{4}\right) \cos (2 \omega t+\phi) .
$$

Spatially resolved measurements 
The set-up used for spatially-resolved measurements is shown on Figure 4a. To measure microparticle speed/position via Doppler velocimetry a weak ( $10 \%$ of the power in the pulsed trapping light) CW probe at $830 \mathrm{~nm}$ was introduced. The different wavelength meant that the trapping light (at 780 to 810 nm) could be blocked away using suitable filters. Photodiode 1 was used to monitor the transmitted power of the pulsed trapping laser and thus to measure the electric field. Photodiode 2 was used to monitor the Doppler signal and measure the speed/position of the particle. The input section of the fibre was placed between two capacitor plates spaced by $2.1 \mathrm{~mm}$ and used to calibrate the response of the sensor to the electric field, i.e., the modulation depth as a function of electric field amplitude. It was important to have the same optical stiffness at the calibration and measurement positions, so that the response of the sensor is identical. This was done by adjusting the power so that the resonant frequency of the trap (related to the optical stiffness) is the same in both the calibration region and the measurement site. Another approach makes use the Langevin force caused by Brownian motion of the gas molecules. By monitoring the resulting spectrum, the trap stiffness can be remotely measured at any particle position, and controlled by adjusting the optical power in the trapping beams. Once the electric field is calibrated and the optical stiffness is maintained at a constant value, an unknown electric field can be measured at any position along the fibre (Figure 4c, bottom).

1. Lee, B. Review of the present status of optical fiber sensors. Opt. Fiber Technol. 9, 57-79 (2003).

2. Willsch, M. Fiber optical sensors in power generation. in Proc. SPIE 8351, 835137-835137-9 (2012).

3. Grattan, K. T. V. \& Sun, T. Fiber optic sensor technology: an overview. Sens. Actuators Phys. 82, 40-61 (2000).

4. Jackson, D. A., Lobo Ribeiro, A. B., Reekie, L. \& Archambault, J. L. Simple multiplexing scheme for a fiber-optic grating sensor network. Opt. Lett. 18, 1192-1194 (1993).

5. Kashyap, R. Fiber Bragg gratings. (Elsevier/Acad. Press, San Diego, 2009).

6. Cruz, J. L. et al. Fibre Bragg gratings tuned and chirped using magnetic fields. Electron. Lett. 33, 235-236 (1997). 
7. Cruz, J. L., Andres, M. V. \& Hernandez, M. A. Faraday effect in standard optical fibers: dispersion of the effective Verdet constant. Appl Opt 35, 922-927 (1996).

8. Laming, R. I. \& Payne, D. N. Electric current sensors employing spun highly birefringent optical fibers. J. Light. Technol. 7, 2084-2094 (1989).

9. Sun, L., Jiang, S. \& Marciante, J. R. All-fiber optical magnetic-field sensor based on Faraday rotation in highly terbium-doped fiber. Opt Express 18, 5407-5412 (2010).

10. Huston, A. . et al. Remote optical fiber dosimetry. Nucl. Instrum. Methods Phys. Res. Sect. B Beam Interact. Mater. At. 184, 55-67 (2001).

11. Bravo, M., Pinto, A. M. R., Lopez-Amo, M., Kobelke, J. \& Schuster, K. High precision microdisplacement fiber sensor through a suspended-core Sagnac interferometer. Opt. Lett. 37, 202-204 (2012).

12. Ashkin, A., Dziedzic, J., Bjorkholm, J. \& Chu, S. Observation of a single-beam gradient force optical trap for dielectric particles. Opt. Lett. 11, 288-290 (1986).

13. Neuman, K. C. \& Block, S. M. Optical trapping. Rev. Sci. Instrum. 75, 2787-2809 (2004).

14. Bishop, A. I., Nieminen, T. A., Heckenberg, N. R. \& Rubinsztein-Dunlop, H. Optical Microrheology Using Rotating Laser-Trapped Particles. Phys Rev Lett 92, 198104 (2004).

15. Yao, A., Tassieri, M., Padgett, M. \& Cooper, J. Microrheology with optical tweezers. Lab Chip 9, 2568-2575 (2009).

16. Michihata, M., Takaya, Y., Miyoshi, T. \& Hayashi, T. Evaluation of laser trapping probe properties for coordinate measurement. in Proc. SPIE 6375, 6375099-10 (2006).

17. Pesce, G. et al. Mapping electric fields generated by microelectrodes using optically trapped charged microspheres. Lab. Chip 11, 4113-4116 (2011).

18. Renn, M. J., Pastel, R. \& Lewandowski, H. J. Laser Guidance and Trapping of Mesoscale Particles in Hollow-Core Optical Fibers. Phys. Rev. Lett. 82, 1574-1577 (1999).

19. Schmidt, O. A., Garbos, M. K., Euser, T. G. \& Russell, P. St.J. Metrology of laser-guided particles in air-filled hollow-core photonic crystal fiber. Opt. Lett. 37, 91-93 (2012). 
20. Euser, T. G., Garbos, M. K., Chen, J. S. Y. \& Russell, P. St.J. Precise balancing of viscous and radiation forces on a particle in liquid-filled photonic bandgap fiber. Opt. Lett. 34, 3674-3676 (2009).

21. Garbos, M. K., Euser, T. G., Schmidt, O. A., Unterkofler, S. \& Russell, P. St.J. Doppler velocimetry on microparticles trapped and propelled by laser light in liquid-filled photonic crystal fiber. Opt. Lett. 36, 2020-2022 (2011).

22. Schmidt, O. A., Euser, T. G. \& Russell, P. St.J. Mode-based microparticle conveyor belt in airfilled hollow-core photonic crystal fiber. Opt. Express 21, 29383-29391 (2013).

23. Benabid, F., Knight, J. \& Russell, P. St.J Particle levitation and guidance in hollow-core photonic crystal fiber. Opt. Express 10, 1195-1203 (2002).

24. Pinto, A. M. R. \& Lopez-Amo, M. Photonic Crystal Fibers for Sensing Applications. J. Sens. 2012, 1-21 (2012).

25. Cubillas, A. M. et al. Photonic crystal fibres for chemical sensing and photochemistry. Chem. Soc. Rev. 42, 8629-8648 (2013).

26. Ashkin, A. Forces of a single-beam gradient laser trap on a dielectric sphere in the ray optics regime. Biophys. J. 61, 569-582 (1992).

27. Hutchins, D. K., Harper, M. H. \& Felder, R. L. Slip Correction Measurements for Solid Spherical Particles by Modulated Dynamic Light Scattering. Aerosol Sci. Technol. 22, 202-218 (1995).

28. Cox, R. G. \& Brenner, H. The slow motion of a sphere through a viscous fluid towards a plane surface-II Small gap widths, including inertial effects. Chem. Eng. Sci. 22, 1753-1777 (1967).

29. Sutherland, W. LII. The viscosity of gases and molecular force. Philos. Mag. Ser. 5 36, 507-531 (1893).

30. White, F. M. Viscous fluid flow. (McGraw-Hill, Boston, 1991).

31. Al Quddus, N., Moussa, W. A. \& Bhattacharjee, S. Motion of a spherical particle in a cylindrical channel using arbitrary Lagrangian-Eulerian method. J. Colloid Interface Sci. 317, 620-630 (2008).

32. Roberts, P. J. et al. Ultimate low loss of hollow-core photonic crystal fibres. Opt. Express 13, 236244 (2005). 
33. Arita, Y., Mazilu, M. \& Dholakia, K. Laser-induced rotation and cooling of a trapped microgyroscope in vacuum. Nat. Commun. 4, 2374 (2013).

34. Gieseler, J., Deutsch, B., Quidant, R. \& Novotny, L. Subkelvin Parametric Feedback Cooling of a Laser-Trapped Nanoparticle. Phys. Rev. Lett. 109, 103603 (2012).

35. Agate, B., Brown, C. T. A., Sibbett, W. \& Dholakia, K. Femtosecond optical tweezers for in-situ control of two-photon fluorescence. Opt. Express 12, 3011-3017 (2004).

\section{Author contributions}

All authors conceived and designed the experiments, D.S.B. performed the experiments. D.S.B., T.G.E and P.St.J.R analysed the data, all authors contributed materials/analysis tools, D.S.B., T.G.E and P.St.J.R wrote the paper.

\section{Figure legends}

Figure 1| Schematic of measurement procedures. a, Measurement set-up and scanning electron micrograph of a HC-PCF (core diameter $12 \mu \mathrm{m}$ ) with a superimposed near-field optical mode profile (at $1064 \mathrm{~nm}$ wavelength). A particle is initially trapped in front of the PCF. By adjusting the power in the forward and backward propagating beams $(\mathrm{P}+, \mathrm{P}-)$ the particle can be moved along the fibre or held stationary. The transmitted power is monitored using a photodiode (PD). Different types of particle-environment interaction can occur in the measurement region: $\mathbf{b}$, An external force acting on the particle, $\mathbf{c}$, a change in the environmental conditions, for example the temperature, or $\mathbf{d}$, a change in particle state, for example, radioluminescence.

Figure 2| Measurements of the particle response at different pressures. a, Typical frequency response of the second harmonic signal plotted versus applied electric field frequency for a particle trapped in HC-PCF and driven by an external electric field (14 mbar, $22 \mathrm{~mW}, 760 \mathrm{~V} / \mathrm{cm})$. The dots are experimental datapoints and the full curves fits to the theory. The inset shows a calibration curve at 6.2 mbar and $22 \mathrm{~mW}$ ( $M$ is the modulation depth). b, Damping coefficients extracted from the theoretical fits, plotted against pressure. The full curve is a theoretical fit. 
Figure 3| Measurement of the particle response at different powers. Frequency spectrum of the response at 6.5 mbar pressure plotted versus applied electric field frequency for two different optical powers (21.2 mW, blue curve with filled-in symbols; $26.6 \mathrm{~mW}$, black curve with open symbols). The applied electric field amplitude was $95 \mathrm{~V} / \mathrm{cm}$. Inset: scaling of the square of the measured resonant frequency ( $\left.f_{\text {res }}\right)$ with the total optical power at the particle position. The slope is $0.4 \mathrm{kHz}^{2} / \mathrm{mW}$.

Figure 4| Spatially resolved measurements of the electric field. a, Schematic of the set-up used for spatially-resolved measurements. b, Cross-section of the patterned electrode (not to scale). The smallest three electrodes have widths $0.05,0.1$ and $0.2 \mathrm{~mm}$ and the gap widths are 10, 5, 3, 2, 1, 0.5 and $0.3 \mathrm{~mm}$. c, Field component normal to the fibre axis, calculated by solving the Helmholtz equation (upper) and flying particle measurement of the field (lower). The red shading represents the estimated error, obtained from the experimentally measured noise level in absence of an external electric field.

Figure 5| Temperature measurements. a, Position-time plot (black line) of the particle as it passes through the reference region $\left(T_{\text {ref }}=293 \mathrm{~K}\right.$ ) and the furnace (at $\left.614 \mathrm{~K}\right)$. The velocity at each point (measured by Doppler velocimetry) is also plotted (light blue curve: the fluctuations are caused by intermodal beating - see text). The solid blue curve is the speed averaged over a moving window 1.3 seconds wide. b, Smoothed speed profile (moving average over a 2-cm-wide window) of the particle with exponential fits (dashed red curves). c, Measured temperature versus set temperature. Error bars were obtained from the standard deviation of the measured microparticle speed. 